\title{
Notch1 Signaling in Pyramidal Neurons Regulates Synaptic Connectivity and Experience-Dependent Modifications of Acuity in the Visual Cortex
}

\author{
Martijn Dahlhaus, ${ }^{1}$ Josephine M. Hermans, ${ }^{1}$ Leonard H. Van Woerden, ${ }^{1,2}$ M. Hadi Saiepour, ${ }^{1}$ Kazu Nakazawa, ${ }^{3}$ \\ Huibert D. Mansvelder, ${ }^{2}$ J. Alexander Heimel, ${ }^{1}$ and Christiaan N. Levelt ${ }^{1}$ \\ ${ }^{1}$ Department of Molecular Visual Plasticity, Netherlands Institute for Neuroscience, an institute of the Royal Netherlands Academy of Arts and Sciences, \\ 1105 BA Amsterdam, The Netherlands, ${ }^{2}$ Department of Experimental Neurophysiology, Center for Neurogenomics and Cognitive Research, VU University \\ Amsterdam, 1081 HV Amsterdam, The Netherlands, and ${ }^{3}$ Genetics of Cognition and Behavior Unit, National Institute of Mental Health, Porter \\ Neuroscience Research Center, Bethesda, Maryland 20892-3710
}

How the visual cortex responds to specific stimuli is strongly influenced by visual experience during development. Monocular deprivation, for example, changes the likelihood of neurons in the visual cortex to respond to input from the deprived eye and reduces its visual acuity. Because these functional changes are accompanied by extensive reorganization of neurite morphology and dendritic spine turnover, genes regulating neuronal morphology are likely to be involved in visual plasticity. In recent years, Notch1 has been shown to mediate contact inhibition of neurite outgrowth in postmitotic neurons and implicated in the pathogenesis of various degenerative diseases of the CNS. Here, we provide the first evidence for the involvement of neuronal Notch1 signaling in synaptic morphology and plasticity in the visual cortex. By making use of the Cre/Lox system, we expressed an active form of Notch1 in cortical pyramidal neurons several weeks after birth. We show that neuronal Notch1 signals reduce dendritic spine and filopodia densities in a cell-autonomous manner and limit long-term potentiation in the visual cortex. After monocular deprivation, these effects of Notch1 activity predominantly affect responses to visual stimuli with higher spatial frequencies. This results in an enhanced effect of monocular deprivation on visual acuity.

Key words: visual; cortex; acuity; plasticity; connectivity; dendritic spine

\section{Introduction}

The primary visual cortex (V1) responds selectively to various features of visual stimuli such as orientation, direction, and spatial frequency (Hübener, 2003). Moreover, most neurons in V1 are more responsive to stimuli from one eye than from the other. How neurons respond in adulthood is strongly influenced by visual experience during postnatal development. When during a critical period of development one eye is deprived, for example, responsiveness of $\mathrm{V} 1$ to the deprived eye decreases, whereas that of the nondeprived eye increases, resulting in a shift of ocular dominance (OD) (Wiesel and Hubel, 1963; Gordon and Stryker, 1996). Monocular deprivation (MD) also counteracts the development of responsiveness to stimuli with high spatial frequencies that occurs during the critical period, thus reducing visual acuity

Received Aug. 19, 2008; accepted Sept. 6, 2008.

This work was supported by a research support grant and a Vidi Grant from the Netherlands Organization for Health Research and Development (ZonMW). H.D.M. and C.N.L. are supported by the Netherlands Academy of Arts and Sciences and ZonMW. J.M.H., J.A.H., L.H.V.W., and C.N.L. are supported by a "Bsik" grant from SenterNovem. We thank Sean Perry, Hans Lodder, Svetlana Škulj, and Sitha Scheltinga for technical assistance, Dr. Susumu Tonegawa for supplying the G35-3-Cre mice, and Dr. Chris de Zeeuw for critical reading of this manuscript.

Correspondence should be addressed to Christiaan N. Levelt, Department of Molecular Visual Plasticity, Netherlands Institute for Neuroscience, an institute of the Royal Netherlands Academy of Arts and Sciences, Meibergdreef 47, 1105 BA Amsterdam, The Netherlands. E-mail: c.levelt@nin.knaw.nl.

DOI:10.1523/JNEUROSCI.1348-08.2008

Copyright $\odot 2008$ Society for Neuroscience ～0270-6474/08/2810794-09\$15.00/0 of the deprived eye (Giffin and Mitchell, 1978; Fagiolini et al., 1994; Prusky and Douglas, 2003). These functional changes are accompanied by extensive structural changes involving growth and retraction of thalamocortical and intracortical axons and turnover of dendritic spines, suggesting that signaling cascades regulating neuronal morphology play an important role in visual plasticity (Shatz and Stryker, 1978; Antonini et al., 1999; Majewska and Sur, 2003; Mataga et al., 2004; Oray et al., 2004; Pizzorusso et al., 2006).

One of these cascades is signaling through the transmembrane receptor Notch1. Although Notch1 (Bray, 2006; Weinmaster and Kopan, 2006) is mostly known for its role in cell-fate decisions during development, it has in recent years been shown to also regulate structural changes in developing neurons. Notch1 is activated after interacting with ligands on neighboring cells, resulting in release of its intracellular domain (NICD) (De Strooper et al., 1999), which together with CBF1/RBP-J $\kappa$ activates transcription of target genes (Lu and Lux, 1996). This cascade stimulates neurite branching but inhibits neurite growth in postmitotic cortical neurons (Berezovska et al., 1999; Sestan et al., 1999; Redmond et al., 2000), neuroblastoma cells (Franklin et al., 1999) and newborn neurons in the dentate gyrus (Breunig et al., 2007). Also the primary transcriptional target of Notch1, hairy and enhancer of split 1 (Hes1), has been shown to restrict neurite outgrowth 
(Jessen et al., 2003). Because Notch1 signaling is upregulated with increasing contacts between neurons in the developing brain (Sestan et al., 1999) and downregulated during a hippocampusdependent learning task (Conboy et al., 2007), Notch1 has been suggested to provide a negative feedback signal controlling neuronal growth and connectivity.

In this study, we used V1 to investigate whether neuronal Notch1 signaling plays a role in plasticity of the mammalian brain. Several studies suggest that it may (Costa et al., 2003; Wang et al., 2004; Costa et al., 2005) but do not exclude the possibility that the observed effects are caused by developmental deficits and/or altered Notch 1 signals in non-neuronal cell types. To circumvent these issues, we used the Cre/Lox system to target expression of constitutively active Notch1 to individual or all pyramidal neurons of the cortex, starting in the fourth postnatal week just before the beginning of the critical period of OD plasticity. Using this approach, we show for the first time that neuronal Notch1 signaling cell-autonomously regulates synaptic connectivity of cortical neurons and affects experience-dependent plasticity in $\mathrm{V} 1$.

\section{Materials and Methods}

DNA constructs and transgenic mice. A construct for Cre-dependent expression of NICD and green fluorescent protein (GFP) was created by cloning cDNA encoding amino acids 1741-2531 of mouse Notch1 into pCDNA3.1 containing the poliovirus internal ribosome entry site (IRES) followed by GFP. The fragment encoding NICD-IRES-GFP was cloned into pThy-lox-stop-lox (LSL) (Chakravarthy et al., 2006), rendering pThyLSL-NICD-IRES-GFP. Transgenic mice were created by pronuclear injections of linearized DNA into fertilized C57BL/6 oocytes. New founders were crossed to the G35-3-Cre line (Sawtell et al., 2003), which had been kept on a C57BL/6 background for at least six generations. Transgenic mice expressing membrane-associated GFP (mGFP) in a Cre-dependent manner have been described previously [line TLG498 (Chakravarthy et al., 2006, 2008)]. All experiments involving mice were approved by the Institutional Animal Care and Use Committee of the Royal Netherlands Academy of Arts and Sciences.

RNA isolation and quantitative PCR. Male mice were anesthetized by intraperitoneal injection of $0.1 \mathrm{ml} / 10 \mathrm{~g}$ bodyweight pentobarbital (Nembutal; Ceva Sante Animale). Occipital cortex was dissected, snap frozen in liquid nitrogen and stored at $-80^{\circ} \mathrm{C}$ until RNA isolation using Trizol (Invitrogen). RNA yield was quantified on a Nanodrop (Isogen Life Science) and quality assessed using an Agilent Bioanalyser. cDNA was synthesized using the SuperScript III First-Strand cDNA Synthesis Kit (Invitrogen). Oligonucleotide primers were designed with Primer Express 2.0 software and purchased from Eurogentec or Biolegio.

Forward and reverse primer sequences for quantitative PCR (qPCR) were as follows: ACTG1-forward (fw), CATTGCTGACAGGATGCAGAA; ACTG1-reverse (rv), ACATCTGCTGGAAGGTGGACA; EF1 $\alpha$ fw, AAGAAGATCGGCTACAACCCAG; EF $1 \alpha$-rv, TTACGCTCTACTTTCCAGCCCT; GAPDH-fw, ATGTGTCCGTCGTGGATCTGA; GAPDH-rv, ATGCCTGCTTCACCACCTTCT; G6PDX-fw, GTCCAGAATCTCATGGTGCTGA; G6PDX-rv, GCAATGTTGTCTCGATTCCAGA; HPRT-fw, GCAAACTTTGCTTTCCCTGG; HPRT-rv, TTCGAGAGGTCCTTTTCACCA; Polr2a - fw, TTTGCGCTGT GTCTGCTTCTT; Polr2a-rv, TGCCCCTTAGATTTGGCCA; Rs27a-fw, GGCCAAGATCCAGGATAAGGA; Rs27a-rv, CCATCTTCCAGCTGCTTACCA; TBP-fw, CACGGACAACTGCGTTGATTT; TBP-rv, GCCCAACTTCTGCACAACTCT; NICD-fw, CGTACTCCGTTACATGCAGCA; NICD-rv, AGGATCAGTGGAGTTGTGCCA; Hes1-fw, TCAACACGACACCGGACAAA; Hes1-rv, CCTTCGCCTCTTCTCCATGAT.

qPCRs were run on the $1 / 20$ diluted cDNA for genes of interest and candidate reference genes. SYBR Green technology was used on a 7300 Real-Time PCR System (Applied Biosystems). Using GeNorm software (http://medgen.ugent.be/ jvdesomp/genorm/), an optimal set of reference genes was chosen based on similar expression patterns across sam- ples (Vandesompele et al., 2002). A geometrical mean of the set was used to normalize results of the genes of interest.

Lentiviral injections. $\mathrm{NICD}^{+} \mathrm{mGFP}^{+}$or $\mathrm{mGFP}^{+}$transgenic males were injected with a lentiviral vector-mediating expression of a CreGFP fusion protein (LV-CreGFP) at postnatal day 22 (P22)-P25. Mice were anesthetized with isoflurane (Abbott). The eyes were protected using artificial tears (Methocel; Novartis Pharma) and patches.

Holes were drilled $2.8 \mathrm{~mm}$ caudal to bregma and $2.5 \mathrm{~mm}$ lateral to the midline. Two hundred nanoliters of LV-CreGFP were injected bilaterally into V1 250-300 $\mu \mathrm{m}$ below the cortical surface using a Nanoject II pump (Drummond Scientific). Mice were analyzed at P38.

Immunohistochemistry. Mice were anesthetized by intraperitoneal injection with pentobarbital and perfused with $4 \%$ paraformaldehyde (Sigma-Aldrich) in PBS. After $2 \mathrm{~h}$ postfixation, $50 \mu \mathrm{m}$ coronal sections of V1 were made using a Leica VT1000S vibratome (Leica). To enhance the EGFP signal and allow long-term storage, free-floating sections were stained using monoclonal mouse anti-GFP (1:500; Millipore Bioscience Research Reagents), followed by goat anti-mouse Cy3 (1:500; Jackson ImmunoResearch). Sections were embedded in Mowiol $[10 \% \mathrm{w} / \mathrm{v}$ Mowiol 4-88 (Merck); 25\% glycerol; and 0.1 $\mathrm{m}$ Tris HCl; pH 8.5] and stored at $4^{\circ} \mathrm{C}$ in the dark until imaging.

For analyzing parvalbumin (PV)-containing boutons surrounding pyramidal neurons, sections were stained using rabbit anti-PV (1:1000; Swant) and mouse anti-NeuN (1:500; Millipore Bioscience Research Reagents), followed by goat anti-rabbit Alexa568 and goat anti-mouse Alexa488 (both 1:500; Invitrogen).

Confocal microscopy and morphological analysis. Cy3-stained mGFPexpressing layer $2 / 3$ pyramidal neurons in V1 were imaged on a Zeiss LSM510 Meta confocal microscope (Zeiss) using a He/ $\mathrm{Ne}(543 \mathrm{~nm})$ laser. Dendritic protrusions on basal dendrites distal to the first branch point were imaged if the distance to either the next branch point or the end of the dendrite was at least $20 \mu \mathrm{m}$. Z-Stacks were made with $200 \mathrm{~nm}$ $Z$-intervals ( $63 \times$ oil objective; numerical aperture 1.4; optical zoom was adjusted for each dendrite). For each neuron of which a dendrite segment was imaged, a single-plane image of the largest cross-sectional area of its soma was acquired ( $63 \times$ objective, $2.5 \times$ optical zoom). An $80 \mu \mathrm{m}$ pinhole was used. Laser intensity, detector gain, and amplifier offset were adjusted for each individual image acquisition to allow use of the entire detector range and avoid saturation. Three-dimensional reconstruction of image stacks was done to facilitate analysis.

Morphological analysis was performed using Zeiss LSM Image Browser (http://www.embl-heidelberg.de/eamnet/html/body_image_ browser.html). Dendritic protrusions of V1 pyramidal neurons expressing mGFP (473 protrusions, 29 dendrites, 26 neurons, 8 mice) or NICD and mGFP (518 protrusions, 39 dendrites, 32 neurons, 10 mice) were analyzed. Spine neck length and the longest straight line in the spine head were determined. Statistical significance of differences in protrusion density, spine neck length, head size, soma size, and densities of filopodia (longer than $2 \mu \mathrm{m}$, no head), mushroom spines (clear neck and head), stubby spines (wider than $0.30 \mu \mathrm{m}$, no neck), and thin spines (no head, shorter than $2 \mu \mathrm{m}$ and a width $<0.30 \mu \mathrm{m}$ ) was determined by Student's $t$ test. At all stages, the investigator was blind to the experimental group.

For analysis of perisomatic inhibitory boutons, PV-containing puncta around NeuN-positive neurons of layer $2 / 3$ cells from V1 were imaged using a $488 \mathrm{~nm}$ argon laser and a $543 \mathrm{~nm} \mathrm{He} / \mathrm{Ne}$ laser. The same settings were used for imaging sections of control and $\mathrm{NICD}^{+} \mathrm{Cre}^{+}$transgenic animals. Single-plane confocal images were converted into TIFF images and analyzed in ImagePro 6 (Media Cybernetics). NeuN-positive cells were marked with a free-drawing tool. Using customized macros, a $2 \mu \mathrm{m}$ ring was drawn around NeuN-positive cells. The number, intensity, and size of PV-puncta within the ring were counted automatically. Analysis was performed blind to genotype. A total of 10 mice were used for the analysis, with five $\mathrm{NICD}^{+} \mathrm{Cre}^{+}$mice $(64$ cells; 221 puncta) and five littermate controls ( 55 cells; 181 puncta).

Slice electrophysiology. Coronal slices (325 $\mu \mathrm{m}$ thickness) of V1 were prepared from 29 - to 34 -d-old $\mathrm{NICD}^{+} \mathrm{Cre}^{+}$male mice (10 slices; 6 animals) or littermate controls (24 slices; 12 animals). Slices were cut in ice-cold slicing artificial CSF (ACSF) containing the following (in $\mathrm{mm}$ ): $125 \mathrm{NaCl}, 3 \mathrm{KCl}, 1.25 \mathrm{NaH}_{2} \mathrm{PO}_{4}, 26 \mathrm{NaHCO}_{3}, 10$ glucose, $3 \mathrm{MgSO}_{4}$, and 
$1 \mathrm{CaCl}_{2}(0.300 \mathrm{mOsm})$ and carboxygenated with $95 \% \mathrm{O}_{2} / 5 \% \mathrm{CO}_{2}$. Slices were allowed to equilibrate for $1 \mathrm{~h}$ at room temperature in carboxygenated ACSF containing the following (in $\mathrm{mm}$ ): $125 \mathrm{NaCl}, 3 \mathrm{KCl}, 1.25$ $\mathrm{NaH}_{2} \mathrm{PO}_{4}, 26 \mathrm{NaHCO}_{3}, 10$ glucose, $1 \mathrm{MgSO}_{4}, 3 \mathrm{CaCl}_{2}$, and 0.01 glycine (300 mOsm).

Slices were put onto a 64-channel multielectrode recording probe (MED64; Alpha MED Sciences) with the electrodes covering layers 1-5 and superfused with $4 \mathrm{ml} / \mathrm{min}$ carboxygenated ACSF at $32^{\circ} \mathrm{C}$. Layer 4 was stimulated with a bipolar extracellular electrode $(0.15 \mathrm{~ms}$; current was adjusted per experiment to evoke half-maximum response), and synaptic response was quantified as the maximum amplitude of the field EPSP (fEPSP) response. After $20 \mathrm{~min}$ baseline recording at $0.1 \mathrm{~Hz}$, long-term potentiation (LTP) was induced by theta burst stimulation (TBS; eight trains of four pulses at $100 \mathrm{~Hz}$, delivered at $200 \mathrm{~ms}$ intervals). TBS was performed three times at 10 s intervals. After LTP induction, fEPSPs were recorded for $40 \mathrm{~min}$ at $0.1 \mathrm{~Hz}$. The number of electrodes in layer $2 / 3$ recording a clear synaptic response was not different between transgenic and control animals (data not shown).

Synaptic responses were analyzed off-line using the recording software Performer 2.0, Igor Pro (WaveMetrics), Excel, and SPSS. Only recordings showing baselines stable within $1.5 \%$ were included. For statistical comparisons, the magnitude of LTP was taken as the average of the $10-40 \mathrm{~min}$ post-TBS time frame. Normality of the distribution was assessed using Kolmogorov-Smirnov testing, followed by two-tailed Student's $t$ test or Kruskal-Wallis analysis. A $p$ value $<0.05$ indicates statistically significant differences. At all stages, the investigator was blind to experimental conditions.

For input/output (I/O) curves, the smallest stimulus size yielding a detectable synaptic response was designated threshold stimulus. Multiples of this stimulus size (generally $10-15 \mu \mathrm{A}$ ) were then used to characterize the input-output relationship. For each stimulus amplitude, six repetitions were performed, yielding an average response size per slice.

In vivo intrinsic signal imaging. Intrinsic signal was imaged transcranially in $62 \mathrm{NICD}^{+} \mathrm{Cre}^{+}$or control mice $\left(\mathrm{NICD}^{+} \mathrm{Cre}^{-}\right.$, $\mathrm{NICD}^{-} \mathrm{Cre}^{+}$, or $\mathrm{NICD}^{-} \mathrm{Cre}^{-}$littermates) as previously described (Heimel et al., 2007). Mice were anesthetized by intraperitoneal injection of urethane $(20 \%$ in saline, $2 \mathrm{~g} / \mathrm{kg}$; Sigma-Aldrich). Heads were fixed and scalps resected. Atropine sulfate $(0.05 \mathrm{mg} / \mathrm{ml}$ in saline, $0.1 \mathrm{mg} / \mathrm{kg}$; Pharmachemie) was injected subcutaneously to reduce mucous excretions. A monitor covered the mice's visual fields from -15 to $75^{\circ}$ horizontally and from -45 to $45^{\circ}$ vertically. The screen was divided into $2 \times 2$ patches, and drifting gratings were used to map the retinotopic representation of V1. The representation of the upper nasal screen patch was used to calculate responses to subsequent tests. For OD measurements, computer-controlled shutters alternated visual stimulation of the eyes using drifting square wave gratings [0.05 cycles per degree (cpd)]. An imaged ocular dominance index (iODI) was defined as iODI $=($ contra response - ipsi response $) /($ contra response + ipsi response). A response to the contralateral eye only corresponds to an iODI of 1 , and $\mathrm{OODI}=-1$ indicates only ipsilateral response. Acuity was determined with $90 \%$ contrast sinusoidal gratings of $0.1,0.2,0.3,0.4,0.5$, and $0.7 \mathrm{cpd}$, phase reversing at $2 \mathrm{~Hz}$ and changing orientation every $0.75 \mathrm{~s}$. A spatial frequency tuning curve was fit to a linear-threshold function using a least-mean-squares procedure. The intersection with zero response strength was our measure of spatial acuity. Student's $t$ tests were used to compute significance levels.

Eyelid suturing. Right eyelids were sutured at P28 under isoflurane anesthesia (Abbott) as previously described (Heimel et al., 2007). At P35 the eyelid was reopened at the start of the imaging session.

Golgi impregnation. Adult (P72-P88), male mice $\left(\mathrm{NICD}^{+} \mathrm{Cre}^{+}\right.$or control) were anesthetized by intraperitoneal injection of a mixture of $0.36 \mathrm{mg} / \mathrm{kg}$ fentanyl citrate, $11.25 \mathrm{mg} / \mathrm{kg}$ fluanisone (sold together as Hypnorm; VetaPharma) and $2.8 \mathrm{mg} / \mathrm{kg}$ midazolam (Dormicum; Roche). The brain was rapidly removed, cut midsagittally, and placed in Golgi-Cox solution ( $1 \%$ potassium dichromate, $1 \%$ mercuric chloride, and $0.8 \%$ potassium chromate in water). The solution was refreshed once. After 3-4 weeks the tissue was dehydrated and embedded in celloidin (Merck).

\section{TLG498}
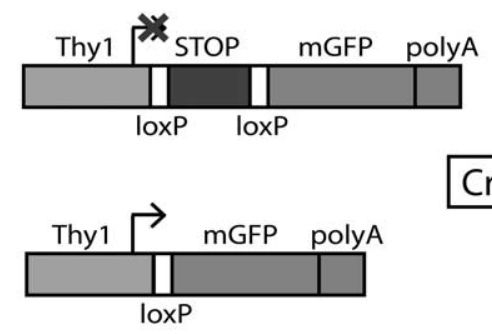

Cre-recombination

\section{TLNic403}

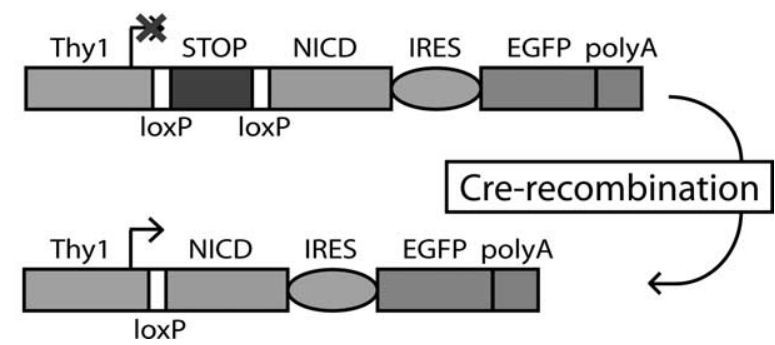

Figure 1. Schematic for mediating Cre-dependent expression of NICD and EGFP in transgenic mice. Mice carry a transgene mediating (re-dependent expression of mGFP (top half; transgenic line TLG498) and/or of NICD and EGFP (bottom half). The NICD transgene (TLNic403) consists of the neuronal Thy1-promoter, followed by a STOP cassette flanked by loxP sites, which inhibits transcription of the adjacent sequence encoding NICD, an internal ribosomal entry site, and EGFP. In the absence of Cre-recombinase, the transcriptional STOP cassette prevents transgene expression. Providing Cre results in excision of the STOP cassette and subsequent activation of transcription.

Coronal sections $(200 \mu \mathrm{m})$ were cut (Reichert Jung Polycut S microtome; Laméris Laboratorium), developed, fixed, dehydrated, and mounted. Slides were coded to render the analyzer blind to experimental condition.

Sholl analysis. Analysis of dendritic branching was done on layer $2 / 3$ pyramidal neurons (controls 42 neurons, 4 mice; $\mathrm{NICD}^{+} \mathrm{Cre}^{+} 17$ neurons, 4 mice). Camera lucida drawings were made using a Zeiss binocular microscope, with a $40 \times$ oil-immersion objective and a camera lucida drawing tube. Selected neurons showed a fully impregnated dendritic tree and spines and no obscuring of branches by blood vessels, precipitate, or other artifacts.

Sholl analysis was performed using an overlay of concentric circles centered on the soma (Sholl, 1953). The number of dendritic intersections crossing each circle (diameters of $25,50,75,100,125,150,200,250$, and $400 \mu \mathrm{m}$ ) was counted.

\section{Results}

Production of transgenic mice expressing NICD in V1 pyramidal neurons

To activate Notch 1 signaling in excitatory neurons of mouse $\mathrm{V} 1$, we produced a mouse line carrying a transgene driving expression of NICD, a constitutively active form of Notch1 (missing the extracellular and transmembrane domains) followed by an IRES and GFP. The transgene contained the Thy 1promoter, restricting expression to neuronal cell types, followed by a "stop-cassette" flanked by loxP sites, rendering it Cre dependent (Fig. 1). Founders were crossed with Cre transgenic line G35-3, expressing Cre-recombinase specifically in all excitatory neurons of cortex and hippocampus (Sawtell et al., 2003). One founder (TLNic403) showed broad and detectable GFP expression in the visual cortex and will be referred to as NICD transgenic mice. In $\mathrm{NICD}^{+} \mathrm{Cre}^{+}$offspring, the transgene was expressed in $>80 \%$ of pyramidal neurons of cortical layers $2 / 3$ and $5 / 6$ (Fig. $2 A$ ), as well as in the dentate 
A

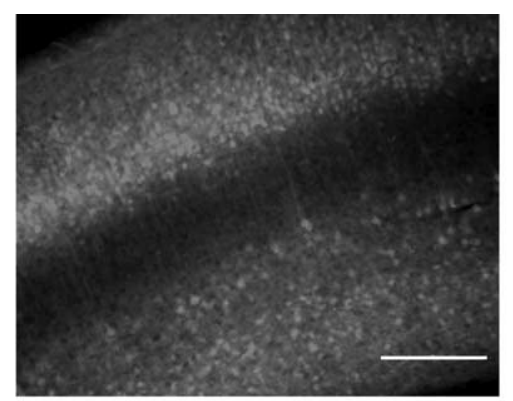

B

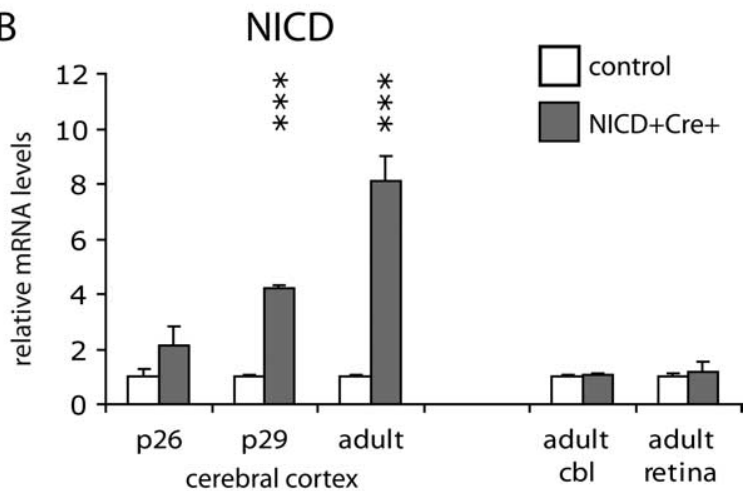

C

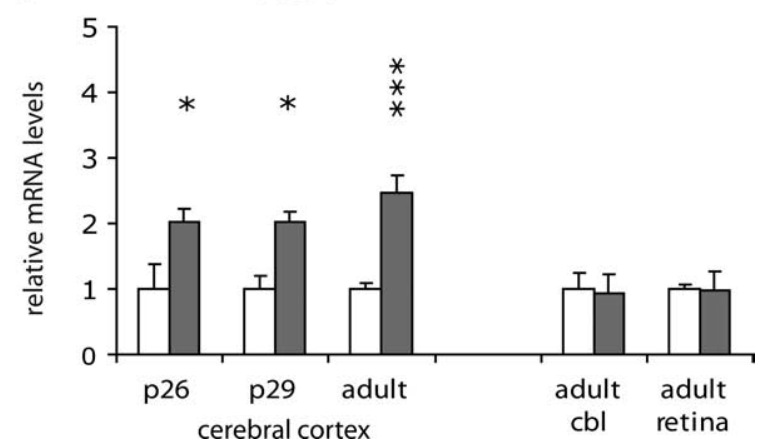

Figure 2. Expression of NICD-IRES-EGFP in visual cortex. $A, \ln \mathrm{NICD}^{+} \mathrm{Cre}^{+}$mice (in this example $61 \mathrm{~d}$ old), the transgene is expressed in pyramidal neurons of cortical layers $2 / 3$ and $5 / 6$. B, Quantification of cortical NICD expression at different ages by qPCR. Transcript levels of $\mathrm{NICD}{ }^{+} \mathrm{Cre}^{+}$animals are plotted relative to age-matched controls. Transgene expression starts during the fourth postnatal week. A lack of increased NICD expression in the adult cerebellum (cbl) confirms that transgene expression requires Cre-recombinase. No transgene expression was detected in the retina. C, Expression of NICD-IRES-EGFP results in increased transcription of Notch1 target genes, as demonstrated by increased Hes $1 \mathrm{mRNA}$ levels. Error bars indicate SEM. ${ }^{*} p<0.05 ;{ }^{* * *} p<0.001$. P26 and P29, 3 controls, 3 transgenics; adult, 19 controls, 22 transgenics. Scale bar, $200 \mu \mathrm{m}$.

gyrus and areas CA1 and CA3. No transgene expression was detected in layer 4, probably because of the lack of Thy1promoter activity in this layer (Fig. $2 A$ ). To confirm that NICD was expressed and functionally active, we used qPCR to analyze expression of NICD and Hes1 (a classical Notch target) at different ages. By P26, expression of Notch1 was twice as high in the cerebral cortex of $\mathrm{NICD}^{+} \mathrm{Cre}^{+}$animals as in control littermates, $4-5$ times as high by P29 and 8 times as high in adults (2-3 months old) (Fig. $2 B$ ). As expected, Credependent transgene expression occurred in cortex but not in the cerebellum or retina (Fig. $2 B$ ). Hesl expression was approximately doubled in the cortex of $\mathrm{NICD}^{+} \mathrm{Cre}^{+}$mice, confirming that the NICD transgene was functional (Fig. 2C).
Notch1 activity cell-autonomously reduces dendritic spine and filopodium density but leaves perisomatic inhibitory boutons unaffected

Because Notch1 has been shown to restrict neuronal growth with increasing neuronal contacts, we asked whether Notch1 also restricts neuronal connectivity at the synaptic level. To study cellautonomous effects of Notch1 on morphology in postmitotic neurons in V1 in vivo, we activated the Cre-dependent NICD transgene by lentivirus-mediated CreGFP expression (LVCreGFP (Ahmed et al., 2004)). Because the level of GFP expression from the NICD(-IRES-GFP) transgene alone was not sufficient for visualizing dendritic protrusions and the GFP of the CreGFP fusion protein was localized to the nucleus, we crossed NICD transgenic animals with mice expressing a Cre-dependent, membrane-associated form of GFP (line TLG498, referred to as mGFP transgenic mice) (Chakravarthy et al., 2006). Bilateral, intracranial LV-CreGFP injections were made in $\mathrm{NICD}^{+} \mathrm{mGFP}^{+}$mice or in $\mathrm{mGFP}^{+}$single transgenic littermates at P22-P25. The resulting Golgi-staining-like transgene expression pattern allowed detailed morphological analyses (Fig. $3 A$ ) of dendritic protrusions from basal dendrites of layer $2 / 3$ pyramidal neurons (Fig. $3 B, C$ ).

NICD expression until $\mathrm{P} 38$ resulted in an $18.6 \%$ reduction in total protrusion density $(16.31 \pm 1.08$ in controls vs $13.28 \pm 0.93$ in transgene expressing cells; $p=0.037$ ) (Fig. $3 D$ ), caused mostly by a $22.0 \%$ reduction in mushroom spine density $(12.83 \pm 0.97$ in controls vs $10.00 \pm 0.82$ in transgene-expressing cells; $p=$ 0.024 ) (Fig. $3 D$ ) and a $59.1 \%$ reduction in filopodial density $(0.69 \pm 0.17$ per $15 \mu \mathrm{m}$ in controls vs $0.28 \pm 0.07$ in NICD transgene expressing cells; $p=0.016$ ) (Fig. 3D). The cross section of the soma was reduced by $8.1 \%$ in NICD-expressing neurons $\left(177.46 \mu \mathrm{m}^{2} \pm 3.42\right.$, vs $193.02 \mu \mathrm{m}^{2} \pm 4.36$ in controls; $p=$ 0.006 ) (Fig. 3E).

No changes were observed in the spine head size between neurons expressing NICD and $\operatorname{mGFP}(0.57 \pm 0.01 \mu \mathrm{m})$ and neurons expressing mGFP only $(0.56 \pm 0.01 \mu \mathrm{m} ; p=0.37)$ (Fig. $3 F)$, nor did NICD expression significantly affect spine neck length $(0.80 \pm 0.03 \mu \mathrm{m}$, vs $0.87 \pm 0.03 \mu \mathrm{m}$ in controls; $p=$ 0.096) (Fig. 3F). Dendrite thickness was not affected by transgene expression (data not shown).

Next, we addressed the question whether increased Notch1 activity in pyramidal neurons also affected the number or size of boutons providing perisomatic inhibitory input. This form of inhibitory input is considered a crucial determinant of plasticity during the critical period, and is provided predominantly by PVexpressing basket cells (Fagiolini et al., 2004). To this end, PVpuncta surrounding layer $2 / 3$ pyramidal neuron somata were analyzed. Sections of broad-expressing 30-d-old transgenic animals (Fig. 2A) and littermate controls were stained for the neuronal nucleus marker NeuN and PV, after which PV-puncta in a ring surrounding the pyramidal neurons were quantified.

The number of PV-puncta per pyramidal cell was not affected by transgene expression $(3.29 \pm 0.27$ in controls versus $3.45 \pm$ 0.31 in transgenics; $p=0.93$ ) (Fig. $3 G$ ), nor was their size, as determined by the percentage of the ring area covered by PVpuncta (controls $3.89 \pm 0.43 \%$; transgenics $3.95 \pm 0.37 \%$; $p=$ 0.87 ) (Fig. $3 G$ ). Punctum intensity was the same between controls and transgenics.

\section{Postsynaptic Notch 1 activity reduces LTP}

The finding that NICD expression reduced spine and filopodia density prompted the question whether NICD expression would also affect LTP induction, which is associated with formation of 

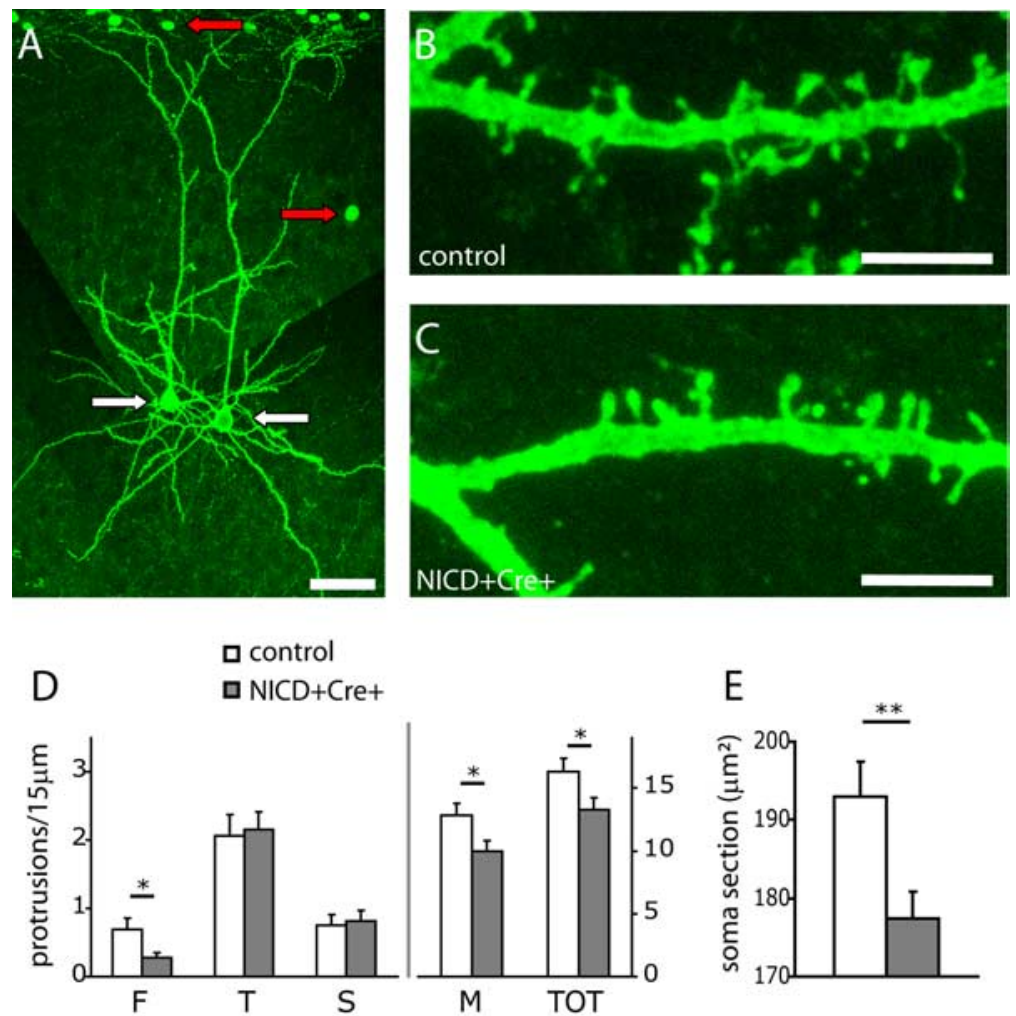

F

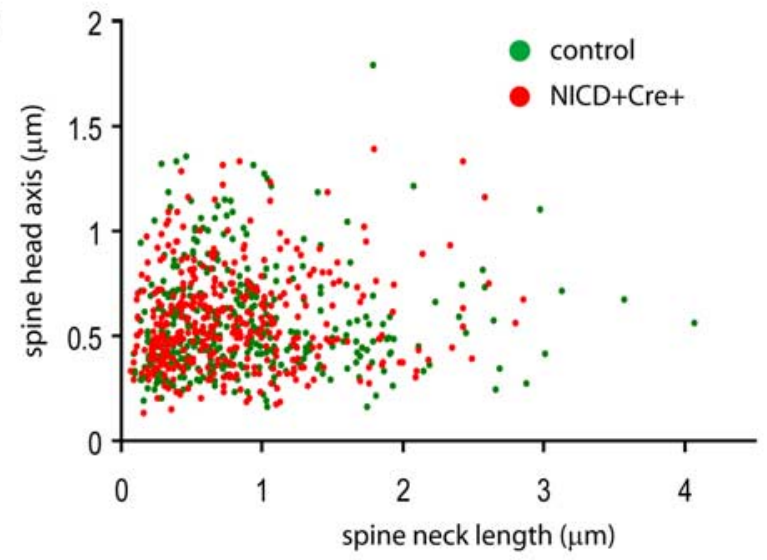

G
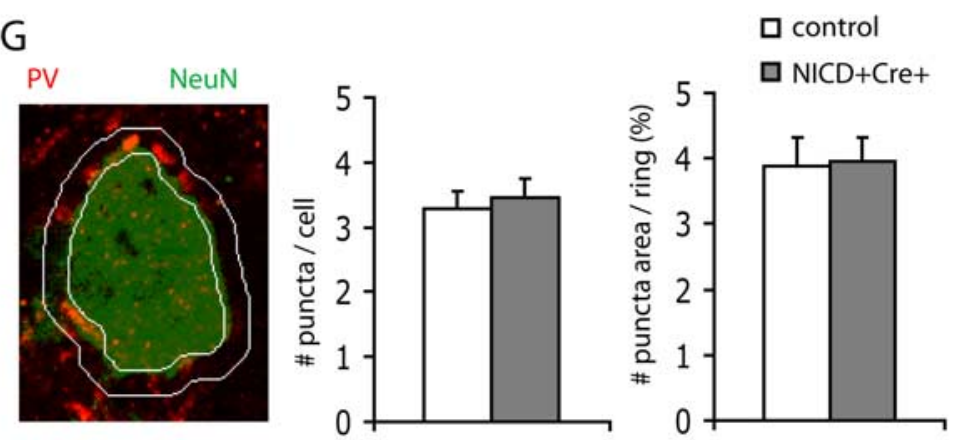

Figure 3. Notch1 activity cell-autonomously reduces spine and filopodium densities in V1, as well as neuronal soma size. $\boldsymbol{A}$, Lentiviral CreGFP-vector injection into V1 results in transduction of neuronal (white arrows) and non-neuronal (red arrows) cells, causing CreGFP expression. The Thy1 promoter is only active in neuronal cells, driving expression of the transgene and mGFP (white arrows). Basal dendrites of layer $2 / 3$ pyramidal neurons were imaged and analyzed. $\boldsymbol{B}, \boldsymbol{C}$, High-magnification projection of confocal images showing basal dendrites of pyramidal neurons expressing mGFP $(\boldsymbol{B})$ or both NICD-IRES-EGFP and mGFP (C). D, Neurons expressing NICD-IRES-GFP have 59\% fewer filopodia than control neurons, 22\% fewer mushroom spines, and a 19\% reduction in total protrusion density. Stubby and thin protrusions are unaffected. $\boldsymbol{E}$, The cross section through the soma of neurons expressing NICD-IRES-EGFP is $8 \%$ smaller than for control neurons. $\boldsymbol{F}$, A correlation plot of spine head diameter and spine neck length shows that in V1, expression of NICD-IRES-EGFP does not affect dendritic spine neck length or spine head size. G, Parval- new spines, probably through filopodia growth and stabilization (Engert and Bonhoeffer, 1999; Maletic-Savatic et al., 1999). LTP induction in connections from layer 4 to layer $2 / 3$ of $\mathrm{V} 1$ was analyzed in $\mathrm{NICD}^{+} \mathrm{Cre}^{+}$mice and control animals.

Synaptic transmission from layer 4 to layer $2 / 3$ was recorded in coronal V1 slices of 29- to 34-d-old mice, before and after induction of LTP by theta-burst stimulation (TBS). In $\mathrm{NICD}^{+} \mathrm{Cre}^{+}$mice, TBS resulted in a smaller increase in synaptic responses $(104.9 \pm 0.9 \%)$ than in littermate controls $(109.3 \pm 1.3 \% ; p=0.045)$ (Fig. $4 C$, example traces in Fig. $4 B$ ). No differences in basic physiology were observed. Neither the I/O curve nor the stimulus strength required for obtaining a halfmaximal response was affected by transgene expression (Fig. 4A). Because in $\mathrm{NICD}^{+} \mathrm{Cre}^{+}$mice, transgenic NICD was expressed in layer $2 / 3$ but not in layer 4 (Fig. $2 \mathrm{~A}$ ), these data indicate that restriction of LTP is caused by increased Notch1 signaling in the postsynaptic neurons.

\section{Notch 1 activity does not affect the} magnitude of ocular dominance plasticity determined using a low spatial frequency stimulus

The reduction in LTP, filopodia formation and total spine numbers suggested that NICD expression in pyramidal neurons reduces their capacity to form new connections. To test whether this had any consequences for OD plasticity in NICD transgenic mice, they were subjected to monocular eyelid suture at the peak of the critical period (P28) and analyzed at P35.

Seven days of MD in control animals resulted in an OD shift, measured by optical imaging of intrinsic signal in the visual cortex using moving square wave grating at $0.05 \mathrm{cpd}$ as a visual stimulus. Examples of ocular dominance maps under the different experimental conditions are shown in Figure $5 A$. After $7 \mathrm{~d}$ of $\mathrm{MD}$, the iODI was reduced in monocularly deprived animals $(0.07 \pm 0.05 ; 6$ animals $)$ compared with nondeprived littermate controls

bumin puncta number and area, representing inhibitory input to the pyramidal neurons, are not different between control animals and NICD transgenic animals. On the left, an example (control) cell is shown with the analysis ring in white indicated around the soma. Green channel intensity has been reduced to facilitate punctum visualization. Error bars indicate SEM. ${ }^{*} p<0.05$; ${ }^{* *} p<0.01 . n=473$ dendritic protrusions for control mGFP-expressing neurons and 518 protrusions for neurons expressing NICD-IRES-EGFP and mGFP. F, Filopodia; T, thin; S, stubby; M, mushroom; TOT, total protrusion numbers. Scale bars: $\boldsymbol{A}, 50 \mu \mathrm{m} ; \boldsymbol{B}, \boldsymbol{C}, 5 \mu \mathrm{m}$. 

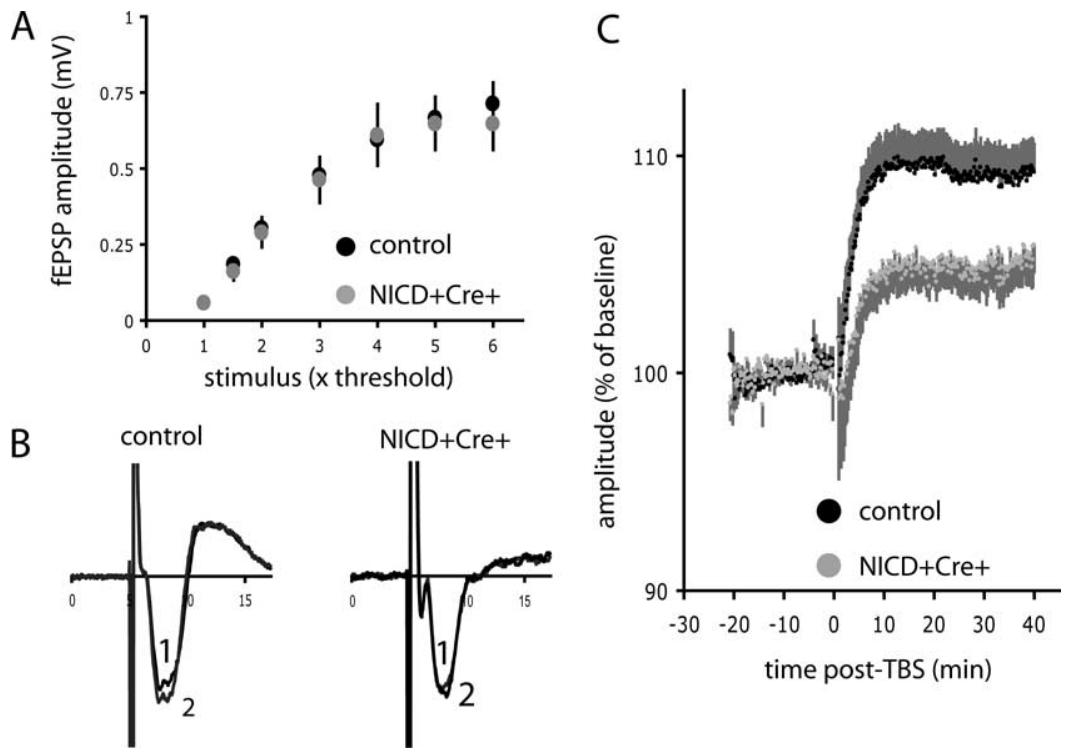

Figure 4. Postsynaptic Notch1 activity reduces LTP. Extracellular stimulation was delivered to layer 4 of V1. Field potentials were recorded in layer 2/3.A, fEPSP amplitude as a function of stimulus intensity (multiples of threshold stimulus). Input- output curves of control and transgenic animals overlap completely. $\boldsymbol{B}$, Example traces of field potentials collected before (1) and after (2) induction of LTP by TBS. Time in milliseconds is indicated along the $x$-axis. C, TBS-induced LTP is reduced in transgenic mice compared with controls $(p<0.05)$. Black circles, Data from controls (24 slices); light gray circles, data from NICD ${ }^{+} \mathrm{Cre}^{+}$ transgenic animals (10 slices); dark gray, error bars indicating SEM.

$(0.35 \pm 0.02 ; n=8$ animals; $p<0.0001)$ (Fig. $5 B)$. This was not different in $\mathrm{NICD}^{+} \mathrm{Cre}^{+}$transgenic animals $[0.31 \pm 0.03$ in nondeprived transgenics $(n=11), 0.04 \pm 0.06$ after $\operatorname{MD}(n=5) ; p=$ 0.001] (Fig. 5B). Interestingly, we noticed that in control animals, the OD shift was caused by both a decrease in deprived eye responses [ $0.74 \pm 0.17$ (value normalized to nondeprived control)] and an increase in open eye responses (1.47 \pm 0.37 ) (Fig. $5 C$ ) [consistent with previous imaging literature (Hofer et al., 2006; Heimel et al., 2008)], whereas in $\mathrm{NICD}^{+} \mathrm{Cre}^{+}$animals the shift seemed to be caused predominantly by decreased responses of the deprived eye $(0.62 \pm 0.10)$, whereas open eye responses remained unchanged (1.05 \pm 0.19$)$ (Fig. 5C). Although the differences between the genotypes were not significant, the observation suggested that more subtle differences in visual responsiveness could be occurring between control and $\mathrm{NICD}^{+} \mathrm{Cre}^{+}$animals after MD.

\section{Notch1 activity increases effects of MD on visual acuity}

Apart from changing OD, MD also counteracts the development of high visual acuity in the deprived eye that normally occurs during the critical period [from $0.3 \mathrm{cpd}$ at P25 to $0.5 \mathrm{cpd}$ at P35 (Heimel et al., 2007)]. The findings that changes in visual acuity could still be induced in adult rats despite the absence of OD plasticity at this age (Iny et al., 2006) and do not always mirror OD in kittens (Murphy and Mitchell, 1986; Faulkner et al., 2006) suggested to us that acuity may be the more sensitive measure for changes in visual responsiveness. We therefore assessed visual acuity in control and $\mathrm{NICD}^{+} \mathrm{Cre}^{+}$animals $7 \mathrm{~d}$ after MD at P35 using optical imaging of intrinsic signals (Heimel et al., 2007). We determined that nondeprived control mice had a spatial acuity of $0.52 \pm 0.02 \mathrm{cpd}$ in the contralateral eye ( $n=3$ animals) (Fig. $6 \mathrm{~A}$ ). MD from P28-P35 resulted in a lower acuity $(0.41 \pm 0.02 \mathrm{cpd}$; $n=8 ; p=0.03)$. In $\mathrm{NICD}^{+} \mathrm{Cre}^{+}$mice, the acuity under control conditions was unaffected $(0.51 \pm 0.05 \mathrm{cpd} ; n=9)$. However, after MD, the acuity was strongly reduced $(0.30 \pm 0.02 \mathrm{cpd} ; n=$ $7 ; p=0.001)$, significantly lower than in deprived control mice $(p=0.004)$ (Fig. 6A). We noticed that although after $\mathrm{MD}, \mathrm{NICD}^{+} \mathrm{Cre}^{+}$mice showed especially weak responses to visual stimuli with the highest spatial frequencies $(0.3-0.4 \mathrm{cpd})$, responses to lower spatial frequency stimuli $(0.1-0.2 \mathrm{cpd})$ were also more affected than in deprived control animals (Fig. 6B). Thus, Notch1 activity caused MD to have a greater impact on responses to visual stimuli with increasing spatial frequencies.

\section{NICD expression does not affect acuity in adult animals}

We next analyzed whether Notch1 activity had similar effects in the adult visual cortex. Like in juvenile animals, we did not see any reduction of acuity in nondeprived adult (2-6 months of age) $\mathrm{NICD}^{+} \mathrm{Cre}^{+}$ mice $(0.52 \pm 0.07 ; n=8)$ compared with controls $(0.53 \pm 0.03 ; n=11)$. However, in contrast to the situation during the critical period, we did not observe differences in the effects of adult MD on visual acuity in $\mathrm{NICD}^{+} \mathrm{Cre}^{+}$mice $(0.39 \pm 0.03 ; n=5$ animals) or control animals $(0.40 \pm 0.04$; $n=6$ ) (Fig. $7 A$ ).

\section{Notch1 signaling does not cause dendritic degeneration in adult V1}

Because it has been proposed that Notch1 activity could cause neuronal degeneration in the adult brain, we assessed whether prolonged NICD expression resulted in any changes in dendritic morphology of pyramidal neurons in adult $\mathrm{NICD}^{+} \mathrm{Cre}^{+}$mice. Brains of 2.5-month-old animals were Golgi-impregnated, after which Sholl analysis was performed on layer $2 / 3$ pyramidal neurons, using coronal slices through V1. The number of intersections of dendrites with respective concentric circles drawn at different distances around the soma was not significantly reduced in $\mathrm{NICD}^{+} \mathrm{Cre}^{+}$mice compared with controls (Fig. $7 B$ ). Also, when all intersections were added up per neuron, the difference between controls $(75.38 \pm 2.84)$ and $\mathrm{NICD}^{+} \mathrm{Cre}^{+}(66.71 \pm 4.87)$ was not significant $(p=0.116)$.

\section{Discussion}

Plasticity in the visual cortex is accompanied by structural rearrangements, including the loss and gain of dendritic spines (Fifková, 1968; Majewska and Sur, 2003; Mataga et al., 2004; Oray et al., 2004). Signaling pathways regulating neuronal morphology are therefore likely to be involved in this form of plasticity. In this respect, the Notch1 signaling pathway is of particular interest. Apart from its well known function in cell-fate decisions and regulation of proliferation and apoptosis, it has also been implicated in limiting neurite outgrowth of postmitotic neurons in the first weeks after birth (Berezovska et al., 1999; Sestan et al., 1999; Redmond et al., 2000). Moreover, various interactions between Notch1 and amyloid precursor protein processing have been observed (Song et al., 1999; Fassa et al., 2005; Fischer et al., 2005), and its expression is increased in Alzheimer's disease (Selkoe, 2001) and other brain disorders (Berezovska et al., 1998; Fischer et al., 2005; Ishikura et al., 2005; Nagarsheth et al., 2006), suggesting that it may play a role in neurodegeneration. Whether neuronal Notch1 signaling regulates synaptic plasticity (Costa et al., 
2005) has remained an unanswered question with important clinical implications. In this study, we addressed this question by analyzing the consequences of increased Notch 1 activity in cortical neurons on ocular dominance plasticity.

We first wanted to establish whether Notch1 activity affected spine density and morphology during the critical period. We found that cell-autonomous activation of Notch1 activity of neurons in the juvenile visual cortex (P23-P38) resulted in a reduction in mushroom spine densities (by $22 \%)$, whereas spine head sizes and neck lengths remained unchanged. In addition, there was a strong reduction in the number of filopodia (by 59\%) after Notch 1 activation. We observed a small reduction in the soma size of neurons expressing active Notch1, which we believe reflects the reduction of total synapse numbers. Because perisomatic inhibitory innervation by $\mathrm{PV}$ expressing basket cells is an important determinant of critical period plasticity, we also determined the size and numbers of PV-positive boutons surrounding pyramidal neurons but detected no differences between NICD transgenic and control mice.

These results show that Notch1 not only restricts dendritic growth during early development, but that it also limits synaptic connectivity at later stages of cortical maturation. Our spine morphological analyses do not allow us to distinguish whether the reduced spine numbers are caused by reduced spine formation or increased spine loss. However, the concurrent reduction in the density of filopodia, which are believed to be precursors of dendritic spines, seems more consistent with the idea that Notch1 activity reduces the potential of neurons to form new excitatory synapses.

We next addressed the question of whether the effect of Notch1 on dendritic spine densities had any consequences for cortical plasticity. Because the induction of LTP has been shown to result in the induction of filopodia (Maletic-Savatic et al., 1999) and spine formation (Engert and Bonhoeffer, 1999), we analyzed whether activation of Notch1 signaling in layer $2 / 3$ pyramidal neurons in V1 reduced the level of LTP that can be induced in the connections between layers 4 and $2 / 3$. We indeed found that postsynaptic NICD expression reduced LTP induction, confirming a functional correlate for the effects of Notch1 on neuronal morphology and providing for the first time direct evidence for a role of neuronal Notch1 signaling in synaptic plasticity.

In apparent contrast to our observation, a previous report has shown that ubiquitous transgenic expression of Notch 1 antisense RNA (NAS) decreased LTP in the hippocampus, whereas addition of the Notch1 ligand Jagged-1 had the opposite effect (Wang et al., 2004). In that report, however, Notch1 signaling was not only altered in pyramidal neurons but in many cell types, and already during development. Because Notch1 signaling in oligodendrocytes interferes with their differentiation (Wang et al., 1998) and affects myelination (Givogri et al., 2002) it seems plau- sible that other (or additional) mechanisms are affected in NAS transgenic animals, explaining the phenotypic difference.

Having confirmed the influence of Notch 1 on neuronal morphology and plasticity, we analyzed whether Notch1 would also affect OD plasticity. To our initial surprise, overexpression of NICD in V1 did not appear to affect the size of the OD shift that is induced by MD. However, further experiments revealed that although visual acuity developed normally in NICD transgenic animals, after MD it was significantly lower than in deprived control animals. This seemed to be caused predominantly by the more strongly reduced responsiveness to the deprived eye in NICD transgenic mice, which became especially prominent with increasing spatial frequency, resulting in a more pronounced reduction of visual acuity after MD. Only because the increase in responsiveness to the nondeprived eye was more apparent in control than in NICD transgenic mice, the relative change in OD was the same in both types of animals.

Thus, neuronal Notch1 activity causes the development or stability of visual acuity to be more dependent on visual experience. What causes this increased vulnerability of the responses to high spatial frequencies in NICD transgenic animals? Currently we can only speculate about this, but the most parsimonious explanation seems to be that although responsiveness to low spatial frequencies is already high before the critical period [and develops even in the absence of experience (Fagiolini et al., 1994)], responsiveness to higher spatial frequencies increases 
A
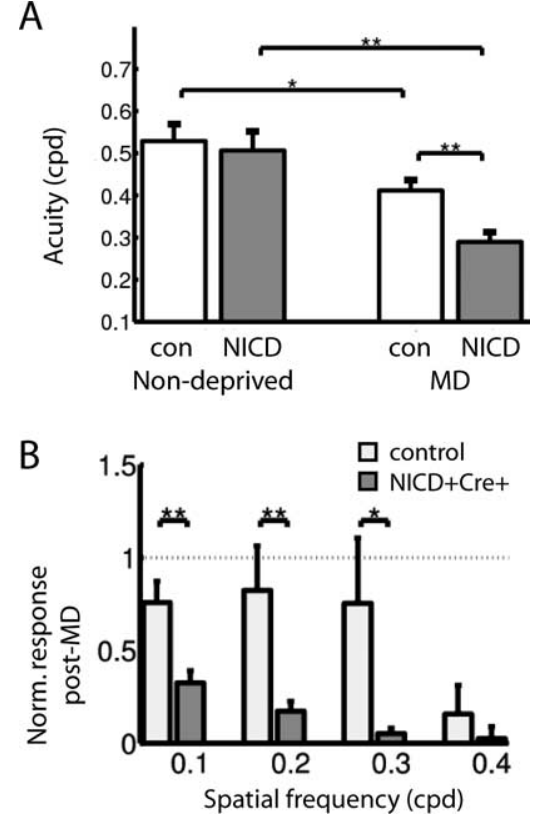

Figure 6. Notch1 activity increases the effects of MD on visual acuity. $\boldsymbol{A}$, Visual acuity in young (P35) animals with or without $7 \mathrm{~d}$ of prior MD. Notch1 activity does not affect acuity in nondeprived mice, but causes enhanced deterioration of visual acuity after MD. B, Deprived-eye response after $M D$, relative to the response in a nondeprived animal. Responsiveness to lowsalience stimuli decreased more in $\mathrm{NICD}{ }^{+} \mathrm{Cre}^{+}$animals after MD, also at lower spatial frequencies. Error bars indicate SEM. ${ }^{*} p<0.05 ;{ }^{* *} p<0.01$. con, Control.

strongly during the critical period (Heimel et al., 2007). The reduced capacity for synaptic strengthening as a result of Notch1 activity could well cause the formation or stability of these developing responses to be more dependent on visual input. This idea is supported by our observation that also an increase in responsiveness to the nondeprived eye does not occur in NICD transgenic animals after MD. This explanation opens the interesting possibility that a relationship between LTP and visual plasticity (Daw et al., 2004) may be found more readily in the development of visual acuity than in altered OD plasticity assessed using optimal visual stimuli.

Our data provide clear evidence for the involvement of neuronal Notch1 signaling in experience-dependent synaptic plasticity during the critical period of OD plasticity, and support the idea that Notch1 signals limit neuronal connectivity. At the same time, we did not obtain convincing evidence for a possible role of Notch1 in dendritic degeneration. Dendritic morphology as assessed with Sholl analysis was not different between adult NICD transgenic animals and controls. We did not find any evidence for a loss in visual performance in adult NICD transgenic mice up to 6 months of age, nor did monocular deprivation during adulthood affect visual acuity more in NICD transgenic animals than in control mice (Fig. 7). Of course, this does not rule out the possibility that Notch1 may have effects on neurite morphology or neuronal maintenance when neurons are at the same time otherwise affected by CNS pathology.

Apart from providing insight into the role of Notch1 in cortical plasticity, our data exemplify how apparently small changes in OD plasticity can have important implications for the development of visual acuity. This stresses the need for studying the mechanisms that underpin plasticity of visual acuity (Giffin and Mitchell, 1978; Murphy and Mitchell, 1986; Fagiolini et al., 1994; Faulkner et al., 2006; Iny et al., 2006; Fischer et al., 2007), espe-
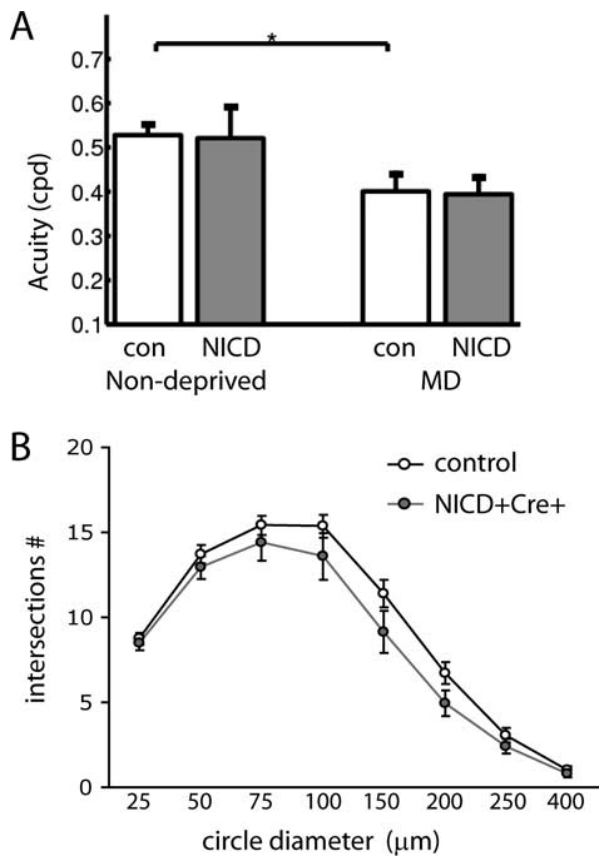

Figure 7. In adult animals, Notch 1 activity does not affect acuity or dendritic morphology. A, Visual acuity in adult animals with or without $7 \mathrm{~d}$ of prior MD. Notch 1 activity does not affect acuity in NICD ${ }^{+} \mathrm{Cre}^{+}$animals, neither in nondeprived animals nor after a week of MD. Error bars indicate SEM. ${ }^{*} p<0.05$. B, Quantitative Sholl analysis of pyramidal neurons in Golgistained adult $\mathrm{V} 1$ of $\mathrm{NICD}{ }^{+} \mathrm{Cre}^{+}$animals ( 4 mice, 17 cells) and controls ( 4 mice, 42 cells). The number of dendritic intersections with eight concentric circles at different distances around the neuronal soma was not affected by prolonged NICD overexpression. Error bars indicate SEM. con, Control.

cially when taking into consideration that loss of acuity is the major problem for people with amblyopia.

\section{References}

Ahmed BY, Chakravarthy S, Eggers R, Hermens WT, Zhang JY, Niclou SP, Levelt C, Sablitzky F, Anderson PN, Lieberman AR, Verhaagen J (2004) Efficient delivery of Cre-recombinase to neurons in vivo and stable transduction of neurons using adeno-associated and lentiviral vectors. BMC Neurosci 5:4.

Antonini A, Fagiolini M, Stryker MP (1999) Anatomical correlates of functional plasticity in mouse visual cortex. J Neurosci 19:4388-4406.

Berezovska O, Xia MQ, Hyman BT (1998) Notch is expressed in adult brain, is coexpressed with presenilin-1, and is altered in Alzheimer disease. J Neuropathol Exp Neurol 57:738-745.

Berezovska O, McLean P, Knowles R, Frosh M, Lu FM, Lux SE, Hyman BT (1999) Notch1 inhibits neurite outgrowth in postmitotic primary neurons. Neuroscience 93:433-439.

Bray SJ (2006) Notch signalling: a simple pathway becomes complex. Nat Rev Mol Cell Biol 7:678-689.

Breunig JJ, Silbereis J, Vaccarino FM, Sestan N, Rakic P (2007) Notch regulates cell fate and dendrite morphology of newborn neurons in the postnatal dentate gyrus. Proc Natl Acad Sci U S A 104:20558-20563.

Chakravarthy S, Saiepour MH, Bence M, Perry S, Hartman R, Couey JJ, Mansvelder HD, Levelt CN (2006) Postsynaptic TrkB signaling has distinct roles in spine maintenance in adult visual cortex and hippocampus. Proc Natl Acad Sci U S A 103:1071-1076.

Chakravarthy S, Keck T, Roelandse M, Hartman R, Jeromin A, Perry S, Hofer SB, Mrsic-Flogel T, Levelt CN (2008) Cre-dependent expression of multiple transgenes in isolated neurons of the adult forebrain. PLoS ONE 3:e3059.

Conboy L, Seymour CM, Monopoli MP, O’Sullivan NC, Murphy KJ, Regan CM (2007) Notch signalling becomes transiently attenuated during long-term memory consolidation in adult Wistar rats. Neurobiol Learn Mem 88:342-351.

Costa RM, Honjo T, Silva AJ (2003) Learning and memory deficits in Notch mutant mice. Curr Biol 13:1348-1354. 
Costa RM, Drew C, Silva AJ (2005) Notch to remember. Trends Neurosci 28:429-435.

Daw N, Rao Y, Wang XF, Fischer Q, Yang Y (2004) LTP and LTD vary with layer in rodent visual cortex. Vision Res 44:3377-3380.

De Strooper B, Annaert W, Cupers P, Saftig P, Craessaerts K, Mumm JS, Schroeter EH, Schrijvers V, Wolfe MS, Ray WJ, Goate A, Kopan R (1999) A presenilin-1-dependent gamma-secretase-like protease mediates release of Notch intracellular domain. Nature 398:518-522.

Engert F, Bonhoeffer T (1999) Dendritic spine changes associated with hippocampal long-term synaptic plasticity. Nature 399:66-70.

Fagiolini M, Pizzorusso T, Berardi N, Domenici L, Maffei L (1994) Functional postnatal development of the rat primary visual cortex and the role of visual experience: dark rearing and monocular deprivation. Vision Res 34:709-720.

Fagiolini M, Fritschy JM, Löw K, Möhler H, Rudolph U, Hensch TK (2004) Specific GABAA circuits for visual cortical plasticity. Science 303:1681-1683.

Fassa A, Mehta P, Efthimiopoulos S (2005) Notch 1 interacts with the amyloid precursor protein in a Numb-independent manner. J Neurosci Res $82: 214-224$.

Faulkner SD, Vorobyov V, Sengpiel F (2006) Visual cortical recovery from reverse occlusion depends on concordant binocular experience. J Neurophysiol 95:1718-1726.

Fifková E (1968) Changes in the visual cortex of rats after unilateral deprivation. Nature 220:379-381.

Fischer DF, van Dijk R, Sluijs JA, Nair SM, Racchi M, Levelt CN, van Leeuwen FW, Hol EM (2005) Activation of the Notch pathway in Down syndrome: cross-talk of Notch and APP. FASEB J 19:1451-1458.

Fischer QS, Graves A, Evans S, Lickey ME, Pham TA (2007) Monocular deprivation in adult mice alters visual acuity and single-unit activity. Learn Mem 14:277-286.

Franklin JL, Berechid BE, Cutting FB, Presente A, Chambers CB, Foltz DR, Ferreira A, Nye JS (1999) Autonomous and non-autonomous regulation of mammalian neurite development by Notch1 and Delta1. Curr Biol 9:1448-1457.

Giffin F, Mitchell DE (1978) The rate of recovery of vision after early monocular deprivation in kittens. J Physiol 274:511-537.

Givogri MI, Costa RM, Schonmann V, Silva AJ, Campagnoni AT, Bongarzone ER (2002) Central nervous system myelination in mice with deficient expression of Notch1 receptor. J Neurosci Res 67:309-320.

Gordon JA, Stryker MP (1996) Experience-dependent plasticity of binocular responses in the primary visual cortex of the mouse. J Neurosci 16:3274-3286.

Heimel JA, Hartman RJ, Hermans JM, Levelt CN (2007) Screening mouse vision with intrinsic signal optical imaging. Eur J Neurosci 25:795-804.

Heimel JA, Hermans JM, Sommeijer JP, Levelt CN (2008) Genetic control of experience-dependent plasticity in the visual cortex. Genes Brain Behav, in press.

Hofer SB, Mrsic-Flogel TD, Bonhoeffer T, Hübener M (2006) Prior experience enhances plasticity in adult visual cortex. Nat Neurosci 9:127-132.

Hübener M (2003) Mouse visual cortex. Curr Opin Neurobiol 13:413-420.

Iny K, Heynen AJ, Sklar E, Bear MF (2006) Bidirectional modifications of visual acuity induced by monocular deprivation in juvenile and adult rats. J Neurosci 26:7368-7374.

Ishikura N, Clever JL, Bouzamondo-Bernstein E, Samayoa E, Prusiner SB, Huang EJ, DeArmond SJ (2005) Notch-1 activation and dendritic atrophy in prion disease. Proc Natl Acad Sci U S A 102:886-891.

Jessen U, Novitskaya V, Walmod PS, Berezin V, Bock E (2003) Neural cell adhesion molecule-mediated neurite outgrowth is repressed by overexpression of HES-1. J Neurosci Res 71:1-6.
Lu FM, Lux SE (1996) Constitutively active human Notch1 binds to the transcription factor $\mathrm{CBF} 1$ and stimulates transcription through a promoter containing a CBF1-responsive element. Proc Natl Acad Sci U S A 93:5663-5667.

Majewska A, Sur M (2003) Motility of dendritic spines in visual cortex in vivo: changes during the critical period and effects of visual deprivation. Proc Natl Acad Sci U S A 100:16024-16029.

Maletic-Savatic M, Malinow R, Svoboda K (1999) Rapid dendritic morphogenesis in CA1 hippocampal dendrites induced by synaptic activity. Science 283:1923-1927.

Mataga N, Mizuguchi Y, Hensch TK (2004) Experience-dependent pruning of dendritic spines in visual cortex by tissue plasminogen activator. Neuron 44:1031-1041.

Murphy KM, Mitchell DE (1986) Bilateral amblyopia after a short period of reverse occlusion in kittens. Nature 323:536-538.

Nagarsheth MH, Viehman A, Lippa SM, Lippa CF (2006) Notch-1 immunoexpression is increased in Alzheimer's and Pick's disease. J Neurol Sci 244:111-116.

Oray S, Majewska A, Sur M (2004) Dendritic spine dynamics are regulated by monocular deprivation and extracellular matrix degradation. Neuron 44:1021-1030.

Pizzorusso T, Medini P, Landi S, Baldini S, Berardi N, Maffei L (2006) Structural and functional recovery from early monocular deprivation in adult rats. Proc Natl Acad Sci U S A 103:8517-8522.

Prusky GT, Douglas RM (2003) Developmental plasticity of mouse visual acuity. Eur J Neurosci 17:167-173.

Redmond L, Oh SR, Hicks C, Weinmaster G, Ghosh A (2000) Nuclear Notch1 signaling and the regulation of dendritic development. Nat Neurosci 3:30-40.

Sawtell NB, Frenkel MY, Philpot BD, Nakazawa K, Tonegawa S, Bear MF (2003) NMDA receptor-dependent ocular dominance plasticity in adult visual cortex. Neuron 38:977-985.

Selkoe DJ (2001) Presenilin, Notch, and the genesis and treatment of Alzheimer's disease. Proc Natl Acad Sci U S A 98:11039-11041.

Sestan N, Artavanis-Tsakonas S, Rakic P (1999) Contact-dependent inhibition of cortical neurite growth mediated by notch signaling. Science 286:741-746.

Shatz CJ, Stryker MP (1978) Ocular dominance in layer IV of the cat's visual cortex and the effects of monocular deprivation. J Physiol 281:267-283.

Sholl DA (1953) Dendritic organization in the neurons of the visual and motor cortices of the cat. J Anat 87:387-406.

Song W, Nadeau P, Yuan M, Yang X, Shen J, Yankner BA (1999) Proteolytic release and nuclear translocation of Notch-1 are induced by presenilin-1 and impaired by pathogenic presenilin-1 mutations. Proc Natl Acad Sci U S A 96:6959-6963.

Vandesompele J, De Preter K, Pattyn F, Poppe B, Van Roy N, De Paepe A, Speleman F (2002) Accurate normalization of real-time quantitative RT-PCR data by geometric averaging of multiple internal control genes. Genome Biol 3:RESEARCH0034.

Wang S, Sdrulla AD, diSibio G, Bush G, Nofziger D, Hicks C, Weinmaster G, Barres BA (1998) Notch receptor activation inhibits oligodendrocyte differentiation. Neuron 21:63-75.

Wang Y, Chan SL, Miele L, Yao PJ, Mackes J, Ingram DK, Mattson MP, Furukawa K (2004) Involvement of Notch signaling in hippocampal synaptic plasticity. Proc Natl Acad Sci U S A 101:9458-9462.

Weinmaster G, Kopan R (2006) A garden of Notch-ly delights. Development 133:3277-3282.

Wiesel TN, Hubel DH (1963) Single-cell responses in striate cortex of kittens deprived of vision in one eye. J Neurophysiol 26:1003-1017. 